\title{
Identification of Critical Biomarkers Responsive to Anti-Autophagy Therapies for Pancreatic Ductal Adenocarcinoma through a Performance Analysis of miRNA Platforms
}

\author{
Sylvia H Sardi ${ }^{* \#}$, Brian J Glassner ${ }^{2 \#}$, J Robert Chang ${ }^{1}$, Sun Hee Yim ${ }^{3}$
}

${ }^{1}$ Laboratory for Innovative and Translational Technologies, Harvard Medical School, 77 Avenue Louis Pasteur, Boston, Massachusetts 02115, USA ${ }^{2}$ WaferGen Biosystems Inc., 7400 Paseo Padre Parkway, Fremont, CA 94555, USA

${ }^{3}$ Division of Genetics, Department of Medicine, Brigham and Women's Hospital, Harvard Medical School, Boston, MA 02115, USA

\#These authors contributed equally to the manuscript

\begin{abstract}
Background: Autophagy is a fundamental catabolic pathway that involves the degradation of unnecessary or dysfunctional cellular components through the lysosomal machinery. The role of autophagy in cancer is complex, exhibiting tumor suppressive or tumor proliferative effects depending on the cancer type. Pancreatic ductal adenocarcinoma has constitutively activated autophagy that results in the selective proliferation of its tumor cells. Understanding the mechanisms by which the inhibition of autophagy occurs could shape our current therapeutic strategies for pancreatic cancer. Recent research has implicated microRNAs as important regulators of autophagy, but how miRNAs influence this inhibition is largely unexplored.
\end{abstract}

Results: It is known that there exists inter-platform variability across miRNA platforms and therefore in order to identify bona fide miRNAs that are involved with the inhibition of autophagy we carried out a performance analysis of miRNA arrays from three vendors. In this paper we report our findings from this cross-platform analysis of miRNA arrays and the identity of several critical biomarkers of response to anti-autophagy therapies in the immortalized human pancreatic ductal adenocarcinoma cells $8988 \mathrm{~T}$ as compared to normal human pancreatic ductal epithelial cells. Our cross-platform analysis of three different miRNA arrays revealed that RT-PCR-based technologies provide higher inter-platform reproducibility than hybridization-based technologies, a greater sensitivity indicated by larger dynamic ranges and reduced processing time. We also discovered two miRNAs, miRNA 720 and miRNA 29b to play a role in the inhibition of autophagy in 8988T cells and found miRNA 30a to be involved with the inhibition of autophagy in normal human pancreatic ductal epithelial cells.

Conclusion: This cross-platform analysis of miRNA arrays contributed significant information about miRNA expression systems and led to the first report of miRNAs implicated in the inhibition of autophagy in pancreatic ductal adenocarcinoma. Our findings should provide new insights for developing therapeutic solutions for this extremely intractable disease.

Keywords: Inhibition of autophagy; microRNA array cross-platform analysis; Pancreatic ductal adenocarcinoma; Chloroquine therapeutic solutions; Qiagen miRNA arrays; NanoString miRNA arrays; WaferGen miRNA arrays

Abbreviations: HPDE: Human Pancreatic Ductal Epithelial Cells; PDAC: Pancreatic Ductal Adenocarcinoma Cells; miRNAs: microRNAs; RT-PCR: Real-Time PCR; $C_{t}$ : Threshold value; $\mathrm{R}^{2}$ : Coefficient of Determination; CV: Coefficient of Variation

\section{Introduction}

Autophagy is a cellular pathway that removes damaged organelles or unused or malformed proteins through the lysosomal machinery and plays a central role in maintaining cell homeostasis. Of the three different forms of autophagy, macro autophagy is the primary pathway and is characterized by the formation of the autophagosome that migrates through the cytoplasm until fusing with a lysosome. The contents of the autophagosome are degraded within the lysosome via acidic lysosomal hydrolases [1]. Autophagy has been shown to be associated with both tumor suppression and tumor cell survival, two opposing processes that can alter the state of an organism [2,3]. Most strategies used to combat cancer recommend inducing autophagy to enhance its tumor suppressive effects, followed by inhibiting autophagy and thereby inducing apoptosis and tumor cell removal. The most effective strategy would be to fine-tune these opposing controls based on the importance of autophagy in a particular cancer or genetic context.
There are over 30,000 cases of pancreatic ductal adenocarcinoma in the US yearly; it is second only to the colon in cancers of the gastrointestinal tract, and is the fourth-leading cause of annual cancer-related deaths. Current treatments are profoundly ineffectiveincidence and mortality rates are essentially equal, and the five year relative survival rate for even local PDACs is only $15 \%$ [4]. PDACs are Ras-driven cancers that have constitutively activated basal autophagy that contributes to continued proliferation of its tumor cells [3]. Understanding how the inhibition of autophagy occurs is crucial for any successful therapeutic intervention in pancreatic cancer. One of the drugs currently available that has been shown to effectively inhibit autophagy in this type of cancer is chloroquine. This

*Corresponding author: Sylvia H Sardi, Harvard Catalyst-Laboratory for Innovative and Translational Technologies, Harvard Medical School, 77 Avenue Louis Pasteur, Boston, Massachusetts 02115, USA, Tel: 617-244-2964; E-mail: shsardi@verizon.net

Received September 18, 2014; Accepted October 30, 2014; Published November 01, 2014

Citation: Sardi SH, Glassner BJ, Chang JR, Yim SH (2014) Identification of Critical Biomarkers Responsive to Anti-Autophagy Therapies for Pancreatic Ductal Adenocarcinoma through a Performance Analysis of miRNA Platforms. J Bioanal Biomed S10: 001. doi:10.4172/1948-593X.S10-001

Copyright: $\odot 2014$ Sardi SH, et al. This is an open-access article distributed under the terms of the Creative Commons Attribution License, which permits unrestricted use, distribution, and reproduction in any medium, provided the original author and source are credited. 
compound blocks lysosomal acidification and hence the last step in the autophagy pathway, autophagosome degradation [5]. The prevalence of autophagy in PDAC coupled with the effectiveness of chloroquine as an anti-autophagy inhibitor may provide a therapeutic solution for a very intractable disease. Chloroquine monotherapy, however, may not be effective [6] and other molecules may need to be combined with chloroquine in order to increase therapeutic efficacy.

In recent years, microRNAs (miRNAs) have emerged as important regulators of a large number of fundamental cellular processes, including autophagy [1]. It has been reported, however, that interplatform variability exists across miRNA platforms [7] and therefore in order to identify bona fide and critical biomarkers responsive to antiautophagy therapies we carried out a performance analysis of miRNA arrays. We chose to utilize current and novel technologies to quantify levels of miRNAs in the inhibition of autophagy. Two platforms were Real-Time PCR (RT-PCR) based, Qiagen and WaferGen, whereas the third was hybridization-based, the NanoString platform. MiRNA levels in normal human pancreatic ductal epithelial cells (HPDE) and immortalized human PDAC cells (line 8988T) with and without chloroquine treatment were analyzed.

\section{Methods}

\section{Sample collection}

Four RNA samples were obtained from human cultured pancreatic cell lines provided by Dr. Alec Kimmelman's laboratory at Harvard Medical School. Samples consisted of a control sample, human pancreatic ductal epithelial cells (HPDE, "Sample A"), and three experimental samples ("Samples B, C, and D"). Sample B consisted of HPDE cells grown in culture media containing the autophagy inhibitor chloroquine $(25 \mathrm{mM})$ for four hours. Samples C and D consisted of immortalized human pancreatic ductal adenocarcinoma cells (PDAC cells, cell line 8988T) grown in untreated media and cell culture media containing $25 \mathrm{mM}$ chloroquine for four hours, respectively [3].

\section{Total RNA extraction and quantification}

Total RNA was extracted from cells using an RNA Extraction Kit (Qiagen, Valencia, CA) following the manufacturer's instructions. RNA sample quality was evaluated using a Bioanalyzer 2100 (Agilent Technologies, Santa Clara, CA) that generates RIN quality scores. RNA concentration was determined using a NanoDrop (Thermo Scientific, Wilmington DE).

\section{Qiagen miRNA expression system}

Three micrograms total RNA from each sample were reverse transcribed with the $\mathrm{RT}^{2}$ miRNA First Strand Kit (Qiagen, Valencia, CA). The resulting cDNA was added to the $2 \mathrm{X} \mathrm{RT}^{2}$ SYBR Green PCR Master Mix (Qiagen, Valencia, CA) and the mixture was aliquotted across the Genome Array Set 384-Well formatted $\mathrm{RT}^{2}$ miRNA PCR Array (Qiagen, Valencia, CA). This array system contains miRNA sequences annotated by the Sanger miRBase Release 14 [8-11]. A total of 376 human miRNA sequences in duplicate were represented in the array. The PCR plate was placed into the Light Cycler 480 (Roche, Indianapolis, IN) and the following cycling program was run - 10 minutes at $95^{\circ} \mathrm{C}$ and 40 cycles of 15 seconds at $95^{\circ} \mathrm{C}, 35$ seconds at $60^{\circ} \mathrm{C}$ and 30 seconds at $72^{\circ} \mathrm{C}$ (Figures 1 and 3 ). In order to obtain statistically significant data, three technical replicates were run for each sample.

\section{WaferGen miRNA expression system}

The technology developed by WaferGen to analyze miRNAs

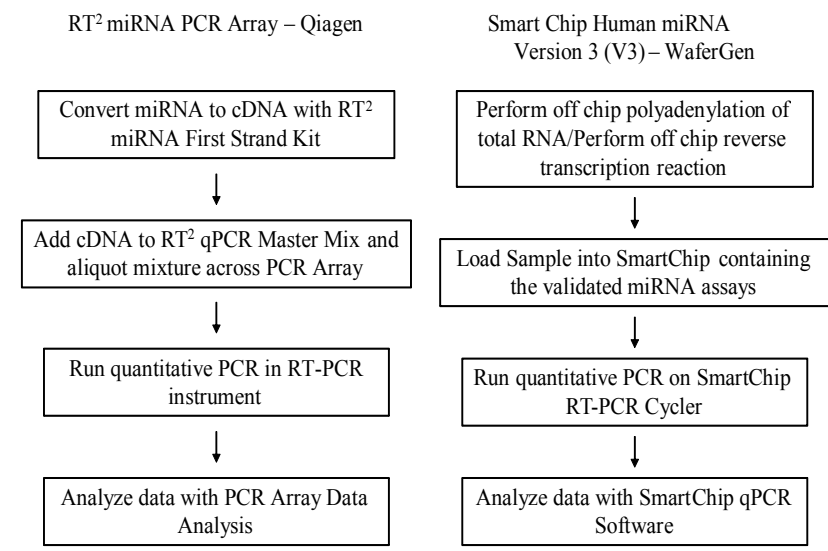

Figure 1: Workflow comparison of RT-PCR-based technologies from Qiagen and WaferGen.

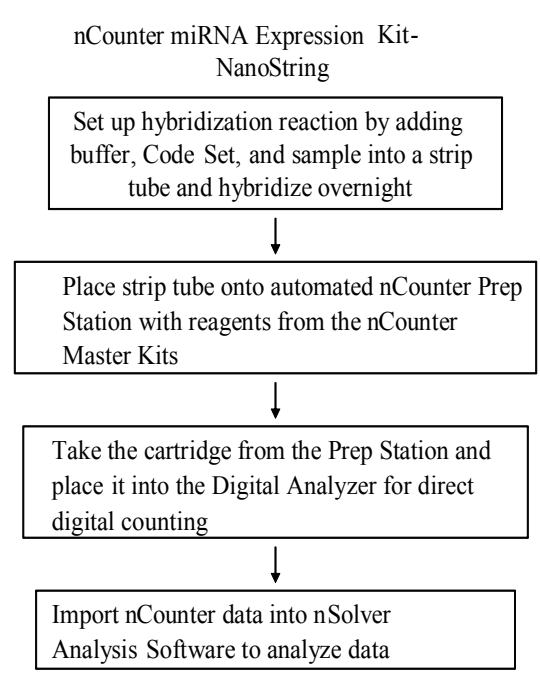

Figure 2: Workflow of the hybridization-based technology from NanoString.

is also RT-PCR based and has the capacity to screen 1,036 human miRNAs in quadruplicate during each run. Two reactions were performed before samples were loaded into the nanowell chip called the SmartChip. The first one was the polyadenylation of total RNA, and the second involved a reverse transcription step using a set of custom primers from WaferGen. Briefly, $1 \mu \mathrm{g}$ of total RNA was polyadenylated using the EpicentrePoly (A) Polymerase Tailing Kit (Epicentre, Madison, WI) and was reverse transcribed using the Applied Biosystems High Capacity cDNA Reverse Transcription Kit (Life Technologies, Carlsbad, CA) following the recommendations of the manufacturer, with the random primer supplied by the kit being replaced with a 20X SmartChip miRNA Reverse Transcription Primer (WaferGen Biosystems, Fremont, CA; final primer concentration $1 \mu \mathrm{M})$. A quantitative PCR reaction using SYBR Green detection was subsequently performed on-chip with preloaded miRNA-specific primers that eliminated the need for pre amplification with complex miRNA primer pools. The cDNA pool was mixed with the Light Cycler 480 SYBR Green I Mix (Life Technologies, Carlsbad, CA) in a $1 \mathrm{ml}$ total final volume and then applied to the SmartChip Human miRNA Panel V3. The SmartChip Panel by WaferGen consists of 1,036 miRNAs from 


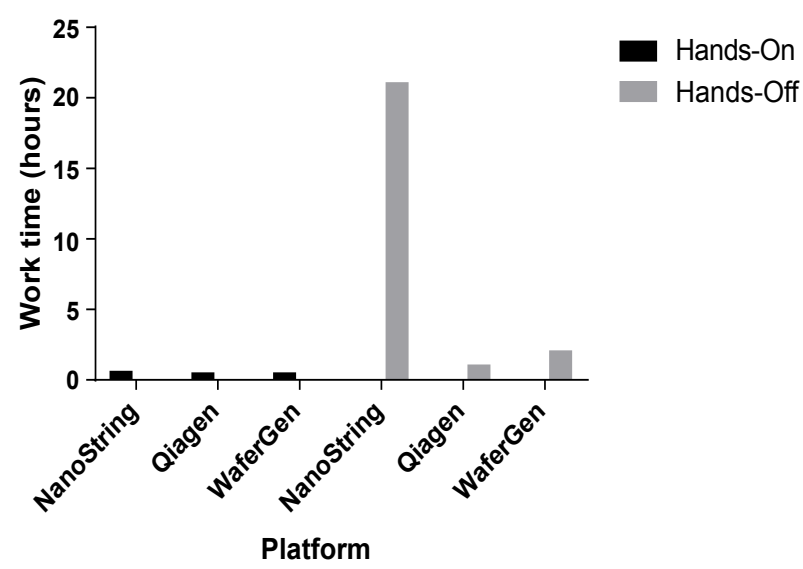

Figure 3: Work time comparison (in hours) of the three miRNA platforms.

the miRBase version 18 sequence database [8-11], in quadruplicate.

The final volume per reaction for the miRNA panel was $100 \mathrm{~nL}$, with an equivalent of $100 \mathrm{pg}$ of starting RNA loaded per reaction. Forty cycles of real-time PCR were performed on the SmartChip Cycler (WaferGen Biosystems, Fremont, CA), followed by a melt curve analysis. The SmartChip Cycler processes the qPCR assays in just over two hours (Figures 1 and 3).

\section{NanoString miRNA expression system}

Buffer and Code Set from the nCounter miRNA Sample Preparation Kit (NanoString, Seattle, WA) and 100ng of total RNA were added into a strip tube and hybridized overnight. This procedure was repeated for each total RNA sample and for three technical replicates. NanoString's hybridization platform for miRNA analysis allows for the screening of 800 human miRNAs with a maximum of forty-eight samples being processed during each run. For the NanoString platform, as miRNAs are released to the miRBase [8-11], new probes can be added to the Code Sets so the data obtained from the NanoString instrument is always current. Each target molecule is represented by a color-coded barcode. The hybridization of barcoded probes to a target molecule occurs through a multiplexed reaction directly in solution. Unique oligonucleotide tags, miR tags, are ligated to mature miRNAs using bridging oligonucleotide and other reagents from the nCounter miRNA Sample Preparation Kit. The bridges are removed using an enzymatic purification step. The mature miR tagged miRNAs are then hybridized to a probe pair - a Reporter Probe that carries the signal and a Capture Probe that allows the probe/target complexes to be immobilized for data collection (Figures 2 and 3 ).

Following hybridization that occurs in a single overnight multiplexed reaction, the strip tube was placed onto the automated nCounter Prep Station (NanoString, Seattle, WA) and reagents from the nCounter Master Kits (NanoString, Seattle, WA) were added according to the nCounter miRNA Expression Assay Kit (NanoString, Seattle, WA) protocol. At this stage, sample processing occurs excess probes are removed and probe/target complexes are bound, immobilized, and aligned on the nCounter Cartridge.

\section{Data analysis}

For the Qiagen platform, after manual collection of the threshold $\left(C_{t}\right)$ values from the real-time PCR machine, the data was entered into the PCR Array Data Analysis Web Portal (Qiagen, Valencia, CA) and automatically analyzed. The fold change for each gene from the control group to the experimental group was calculated as $2^{-\Delta \Delta \mathrm{Ct}}[12]$.

For the WaferGen platform, relative miRNA expression was determined by the data obtained with the real-time instrument and the $\Delta \Delta \mathrm{C}_{t}$ method as for the Qiagen system. Both raw $\mathrm{C}_{t}$ and $\mathrm{T}_{\mathrm{m}}$ values for each assay and sample were collected for data analysis. A data quality screen based on amplification, $\mathrm{T}_{\mathrm{m}}$ values from melt curves, and $\mathrm{C}_{\mathrm{t}}$ and $\mathrm{T}_{\mathrm{m}}$ variability was performed to remove any outlier data before $\Delta \Delta \mathrm{C}_{\mathrm{t}}$ calculations were used to determine fold change in miRNA levels.

The mean $\mathrm{C}_{t}$ and $\mathrm{T}_{\mathrm{m}}$ values determined from each of the 4 replicates on each SmartChip Panel were calculated by the SmartChip qPCR Software (WaferGen Biosystems, Fremont, CA) for each assay and reported (minus any discarded outlier assays). These replicate data were exported to Excel (Microsoft, Redmond, WA). For the sake of ease of computation, assays for which no $C_{t}$ value was reported or for $\mathrm{C}_{t}$ values $>30.0$, a $\mathrm{C}_{t}$ value of 30 was assigned (i.e., miRNAs absent or below the level of detection). $\Delta \mathrm{C}_{t}$ values were computed using the mean of all replicate $C_{t}$ values for each sample/chip, not including $C_{t} s$ of 30 [13]. The average $\Delta C_{t}$ for the experimental samples (B-D) and the control sample A for each assay were then computed. From these, $\Delta \Delta \mathrm{C}_{\mathrm{t}}$ s were computed $\left(\Delta \mathrm{C}_{\mathrm{t}}\right.$ Experimental $-\Delta \mathrm{C}_{\mathrm{t}}$ Control $)$.

For the NanoString platform, sample nCounter cartridges (NanoString, Seattle, WA) were placed into the Digital Analyzer (NanoString, Seattle, WA), a fluorescent reader, for direct digital counting. The barcodes can be individually counted without the need for amplification providing very sensitive digital data. The nCounter data was imported and analyzed in nSolver Analysis Software (NanoString, Seattle, WA)

For all three platforms, differences in the miRNA levels of samples B-D were expressed as fold-changes relative to the levels detected in sample A. Because all assays were reported in triplicate (except for the WaferGen assays which were reported in quadruplicate), mean values were computed prior to fold-change calculations. For the purpose of comparison between the different platforms, miRNA levels that differed by more than 2 -fold (increased or decreased) relative to the control sample A levels were considered to be significant. In order to control the variability among experiments, a set of endogenous control miRNAs were incorporated into each miRNA expression system.

\section{Statistical analysis}

The coefficients of determination $\mathrm{R}^{2}$ were calculated for the intraplatform variation analysis. Individual hybridization values obtained from the NanoString platform and individual $C_{t}$ values from the Qiagen and WaferGen platforms were compared between triplicate samples. The $\% \mathrm{CV}$ is defined as the variation among multiple measurements in proportion to their mean. We calculated \%CV for the inter-platform variation analysis. Average hybridization values obtained from the NanoString platform and average $C_{t}$ values from the Qiagen and WaferGen platforms were compared among platforms for the 26 assays in common to all of them.

\section{Results}

\section{Workflow comparison of miRNA expression systems from three vendors}

In our cross-platform analysis we first compared the workflow of the miRNA expression systems from three vendors. In the first sample 
processing step, the two real-time based assay systems from Qiagen and WaferGen followed very similar workflows (Figure 1). Both systems converted the miRNA species to cDNA with the aid of their respective reverse transcription kits. Samples containing the cDNA were then mixed with the validated miRNA assays and quantitative PCR reactions were run in the respective system instruments. Lastly, the data was analyzed with each vendor's proprietary software. For the hybridization based assay system from NanoString, the workflow was entirely different (Figure 2). Hybridization reactions were set up by adding sample, buffer and barcoded probes to a strip tube and hybridized overnight. The strip tube was then transferred to a station where sample processing occurs and probe/target complexes are bound to a cartridge. This cartridge was then placed into another instrument for direct digital counting. The resulting data was analyzed by NanoString's proprietary software.

Several other parameters related to workflow were then compared including sample processing times, accessibility of the platforms and extent of high-throughput screening. A comparison of the three workflows revealed that the real-time based technologies from Qiagen and WaferGen have reduced sample processing times compared with NanoString's hybridization based platform which requires an overnight hybridization step (Figure 3). Also, Qiagen's miRNA expression system which can be run on a standard PCR machine is the most cost effective and therefore the most accessible of the three platforms. NanoString and WaferGen's miRNA expression systems require more elaborate technology platforms for sample processing and therefore can be inhibitive for many researchers. In regards to high-throughput screening, all three miRNA expression systems have this capability. WaferGen's miRNA platform offers the largest number of miRNAs, over 1,000 that can be screened during a single run. For the Qiagen platform, 376 human miRNAs can be screened during a single run. The NanoString platform offers an intermediate number of screenable miRNAs, approximately 800 compared to the other two platforms. The advantage of the NanoString platform compared with the other two platforms is that 48 experimental samples can be screened for miRNA expression during a single run instead of only a single sample.

\section{Comparison of fold-change data for two platforms}

In order to understand the meaning of the fold-change values better, comparison of the fold-change data common to the Qiagen and WaferGen platforms and the WaferGen and NanoString platforms were analyzed separately. A total of 50 miRNAs were common to the Qiagen and WaferGen platforms that displayed at least a 2-fold increase in miRNA levels as compared to the control sample A (Table 1). Approximately 20 miRNA assays that were common to the Qiagen and WaferGen platforms displayed at least a two-fold decrease in miRNA levels as compared to the control sample A (Table 1).

When comparing the fold-data generated by the WaferGen platform to the fold-data generated by the NanoString platform, 23 miRNA assays displayed at least a 2 -fold increase in miRNA levels as compared to the control sample A (Table 1). A two-fold decrease

\begin{tabular}{|l|c|c|c|}
\hline Platforms Compared & $\mathbf{2}$ & $\mathbf{2}$ & $\mathbf{2} \mathbf{2}$ or $\leq \mathbf{2}$ \\
\hline NanoString/WaferGen & 23 & 8 & N/A \\
\hline Qiagen/WaferGen & 50 & 20 & N/A \\
\hline NanoString/Qiagen/WaferGen & N/A & N/A & 26 \\
\hline
\end{tabular}

Fold-change Data Totals

Table 1: Comparison of the fold-change data for two platforms and three platforms that displayed at least a 2-fold increase, 2-fold decrease or a combination of both relative to the control sample. in miRNA levels as compared to the control sample A was observed for only 8 miRNA assays common to both WaferGen and NanoString (Table 1).

\section{Intra-platform variation analysis}

A comparison of individual data points among replicates for which there were at least three informative assays for the control sample A was performed for each platform. For the NanoString platform, coefficients of determination $\left(\mathrm{R}^{2}\right)$ values were calculated for hybridization values from triplicate samples. A $\mathrm{R}^{2}$ value of 0.9804 was obtained for the NanoString platform from a variation analysis of 121 assays (Figure 4). The $\mathrm{R}^{2}$ value for triplicate samples from 744 assays run on the Qiagen platform was 0.9152 (Figure 4). A similar $\mathrm{R}^{2}$ value was obtained with WaferGen platform. A $\mathrm{R}^{2}$ value of 0.9116 was calculated for triplicate samples of 1004 assays analyzed with the WaferGen platform (Figure $4)$.

\section{Inter-platform variation analysis}

Among all of the assays employed, there were 26 miRNA assays overall that were common to all three platforms and displayed a significant difference relative to the control sample A (Table 2). Although the direction and magnitude of gene expression were similar for all 26 assays, the variability among the three platforms differed significantly. The NanoString platform exhibited the highest variability among the three, with a $\% \mathrm{CV}$ at $17.1 \%$ (Table 3). The WaferGen platform demonstrated the best reproducibility with a $\% \mathrm{CV}$ at $0.5 \%$ (Table 3). An intermediate reproducibility value was calculated for the Qiagen platform at 4.3\% (Table 3).

\section{Dynamic range variations evident among the platforms}

The dynamic range for all three platforms was also significantly different. There were 46 assays from the NanoString platform that displayed a greater than 2 -fold difference in miRNAs compared to the control sample. Fold changes for all assays on this platform ranged from a value of 0 to 6.5 -fold (Table 4). WaferGen had the greatest number of assays, a total of 262 , that displayed a greater than 2 -fold difference in miRNA levels compared to the control sample. Fold changes for all assays on this platform extended from 0 to 10,420-fold (Table 4). The Qiagen platform had a total of 170 assays that displayed a greater than 2 -fold difference in miRNA levels in the experimental samples relative to the control sample. The range of fold changes for all assays on this platform was intermediate to NanoString and WaferGen's, extending from 0 to 50.9 -fold (Table 4).

\section{Cross-platform determination of miRNA levels in HPDE and 8988T cells treated with the autophagy inhibitor chloroquine}

In this study, we evaluated miRNA expression systems from three vendors by screening miRNAs involved with the inhibition of autophagy in immortalized human PDAC cells, cell line 8988T, as compared to normal human pancreatic ductal epithelial cells designated as HPDE cells. Analysis of the cross-platform miRNA data revealed two miRNAs to be significantly up-regulated in the $8988 \mathrm{~T}$ cells grown in the presence of the autophagy inhibitor chloroquine. These miRNAs are miR-29b and the miR-720. Based on the NanoString platform miRNA data, miR-29b in $8988 \mathrm{~T}$ cells exposed to chloroquine exhibited a 2.24 fold increase in expression relative to miR-29b levels exhibited by untreated 8988T cells (Table 5). Under the same experimental conditions but utilizing the Qiagen platform, a similar 2.46 fold induction of miR-29b expression was exhibited in chloroquine treated $8988 \mathrm{~T}$ cells compared to untreated cells (Table 5). In contrast, no significant induction of 
Citation: Sardi SH, Glassner BJ, Chang JR, Yim SH (2014) Identification of Critical Biomarkers Responsive to Anti-Autophagy Therapies for Pancreatic Ductal Adenocarcinoma through a Performance Analysis of miRNA Platforms. J Bioanal Biomed S10: 001. doi:10.4172/1948-593X.S10-001
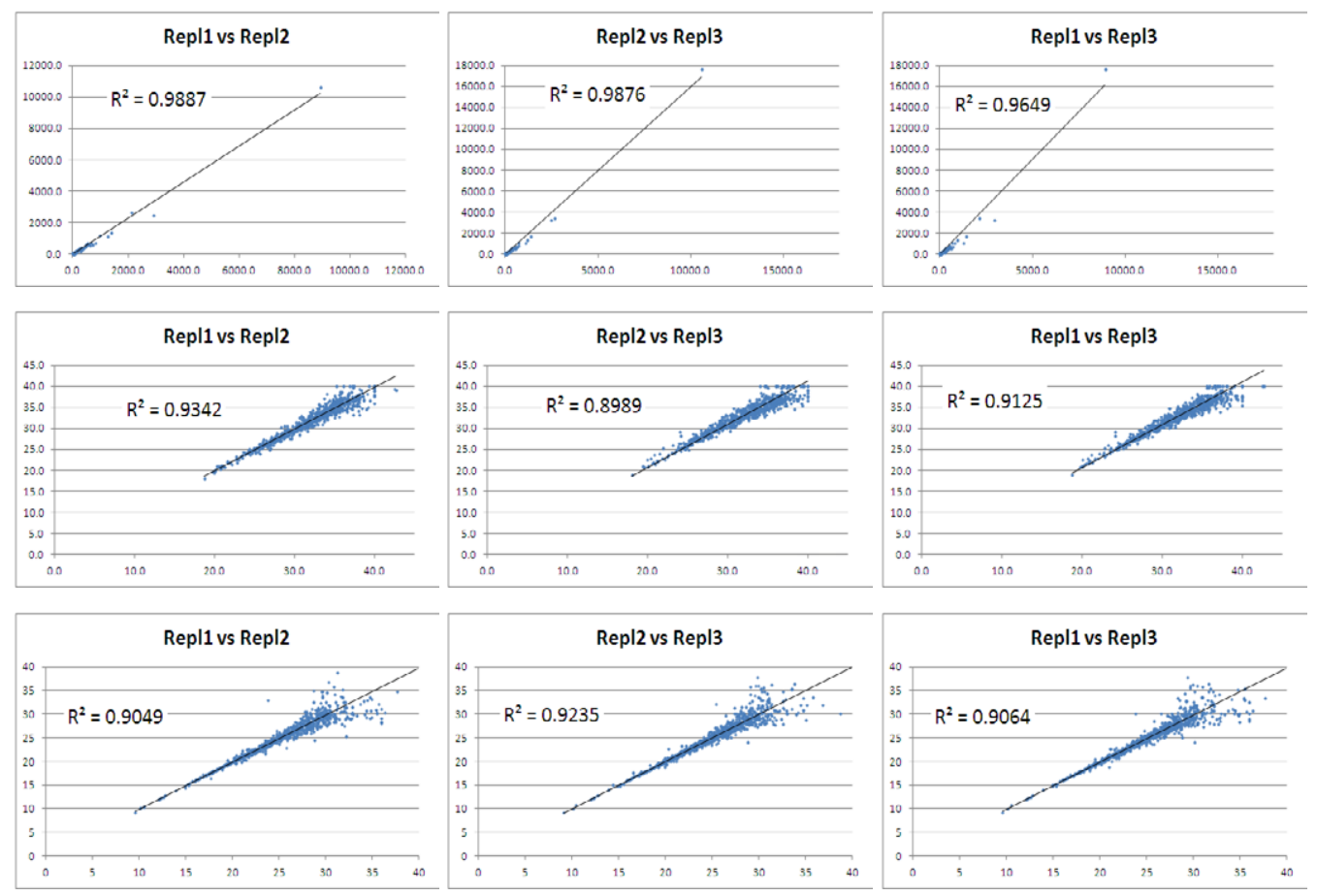

NanoString

$\mathrm{n}=121$

assays

$R_{\text {mean }}^{2}=0.9804$

Qiagen

$n=744$

assays

$\mathrm{R}_{\text {mean }}^{2}=0.9152$

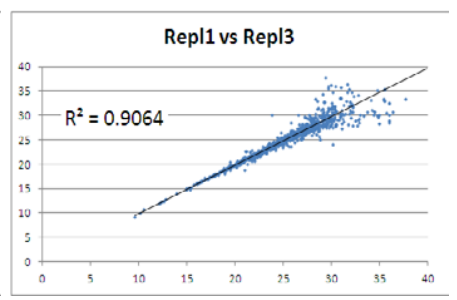

WaferGen

$\mathrm{n}=1,004$

assays

$R_{\text {mean }}^{2}=0.9116$

Figure 4: Intra-platform variation analysis. Comparison of individual hybridization values (NanoString) and $C_{t}$ values (Qiagen and WaferGen) for each platform among replicates for which there were at least three informative assays for the control sample A.

\begin{tabular}{|c|c|c|c|c|c|c|c|c|c|c|c|c|}
\hline \multirow[t]{2}{*}{ miRNA Assays } & \multicolumn{4}{|c|}{ NanoString Platform } & \multicolumn{4}{|c|}{ Qiagen Platform } & \multicolumn{4}{|c|}{ WaferGen Platform } \\
\hline & A/A & $\mathrm{B} / \mathrm{A}$ & C/A & D/A & A/A & $\mathrm{B} / \mathrm{A}$ & C/A & D/A & A/A & $B / A$ & C/A & D/A \\
\hline HSA-let-7d & 1 & 0.85 & 3.02 & 2.29 & 1.00 & 1.37 & 5.06 & 5.58 & 1.00 & 1.02 & 2.48 & 2.59 \\
\hline HSA-let-7g & 1 & 0.99 & 7.18 & 5.78 & 1.00 & 0.78 & 9.34 & 10.75 & 1.00 & 1.13 & 4.11 & 4.49 \\
\hline HSA-let-7i & 1 & 1.03 & 4.27 & 2.97 & 1.00 & 0.86 & 4.78 & 5.03 & 1.00 & 1.03 & 3.20 & 3.17 \\
\hline HSA-MIR-100 & 1 & 1.05 & 2.89 & 2.57 & 1.00 & 0.85 & 4.30 & 3.61 & 1.00 & 0.96 & 2.74 & 3.17 \\
\hline HSA-MIR-10a & 1 & 1.13 & 8.61 & 6.29 & 1.00 & 1.18 & 58.49 & 50.91 & 1.00 & 0.97 & 3.99 & 4.51 \\
\hline HSA-MIR-126 & 1 & 0.92 & 4.08 & 3.56 & 1.00 & 0.86 & 4.68 & 3.63 & 1.00 & 0.98 & 4.30 & 4.68 \\
\hline HSA-MIR-140 & 1 & 1.13 & 1.96 & 2.63 & 1.00 & 0.94 & 7.38 & 7.82 & 1.00 & 1.18 & 5.85 & 6.59 \\
\hline HSA-MIR-151 & 1 & 1.41 & 4.85 & 5.61 & 1.00 & 0.80 & 4.20 & 2.48 & 1.00 & 0.93 & 3.58 & 3.40 \\
\hline HSA-MIR-15a & 1 & 0.88 & 7.62 & 6.43 & 1.00 & 0.82 & 7.31 & 9.74 & 1.00 & 0.92 & 8.01 & 7.74 \\
\hline HSA-MIR-15b & 1 & 0.87 & 2.61 & 2.46 & 1.00 & 1.17 & 2.84 & 4.02 & 1.00 & 1.07 & 2.47 & 2.78 \\
\hline HSA-MIR-16 & 1 & 1.05 & 3.78 & 4.02 & 1.00 & 0.87 & 3.54 & 2.58 & 1.00 & 0.98 & 3.76 & 4.03 \\
\hline HSA-MIR-191 & 1 & 1.23 & 3.31 & 2.40 & 1.00 & 0.92 & 4.50 & 3.54 & 1.00 & 1.08 & 3.07 & 3.14 \\
\hline HSA-MIR-200c & 1 & 1.10 & 0.01 & 0.01 & 1.00 & 0.62 & 0.00 & 0.00 & 1.00 & 1.00 & 0.00 & 0.00 \\
\hline HSA-MIR-205 & 1 & 0.82 & 0.00 & 0.00 & 1.00 & 0.77 & 0.00 & 0.00 & 1.00 & 0.97 & 0.00 & 0.00 \\
\hline HSA-MIR-29a & 1 & 1.12 & 3.80 & 3.54 & 1.00 & 0.79 & 4.23 & 4.26 & 1.00 & 1.02 & 2.89 & 3.63 \\
\hline HSA-MIR-29b & 1 & 1.09 & 2.28 & 5.12 & 1.00 & 0.93 & 3.93 & 9.65 & 1.00 & 0.79 & 9.07 & 9.39 \\
\hline HSA-MIR-29c & 1 & 0.84 & 2.70 & 2.87 & 1.00 & 0.83 & 4.17 & 4.33 & 1.00 & 0.81 & 2.92 & 3.57 \\
\hline HSA-MIR-30a & 1 & 0.64 & 4.71 & 5.11 & 1.00 & 0.66 & 4.30 & 4.91 & 1.00 & 0.76 & 5.04 & 5.12 \\
\hline HSA-MIR-30b & 1 & 1.05 & 3.83 & 4.48 & 1.00 & 0.64 & 4.54 & 3.51 & 1.00 & 0.99 & 4.91 & 5.33 \\
\hline HSA-MIR-31 & 1 & 1.10 & 0.09 & 0.09 & 1.00 & 0.47 & 0.03 & 0.03 & 1.00 & 0.90 & 0.02 & 0.02 \\
\hline HSA-MIR-361 & 1 & 0.94 & 2.09 & 2.29 & 1.00 & 0.82 & 4.96 & 5.36 & 1.00 & 0.90 & 4.36 & 5.49 \\
\hline HSA-MIR-363 & 1 & 0.81 & 0.29 & 0.23 & 1.00 & 0.83 & 0.15 & 0.12 & 1.00 & 0.92 & 0.01 & 0.01 \\
\hline HSA-MIR-720 & 1 & 1.71 & 2.12 & 2.85 & 1.00 & 0.92 & 1.61 & 5.02 & 1.00 & 1.09 & 0.45 & 0.98 \\
\hline HSA-MIR-9 & 1 & 0.98 & 0.06 & 0.06 & 1.00 & 0.66 & 0.03 & 0.02 & 1.00 & 1.08 & 0.01 & 0.00 \\
\hline HSA-MIR-98 & 1 & 1.11 & 2.60 & 2.63 & 1.00 & 0.93 & 2.17 & 2.86 & 1.00 & 1.08 & 2.31 & 2.28 \\
\hline HSA-MIR-99a & 1 & 1.18 & 0.55 & 0.40 & 1.00 & 0.80 & 0.29 & 0.25 & 1.00 & 1.02 & 2.32 & 2.77 \\
\hline
\end{tabular}

Fold-change Data for miRNAs Common to all Three Platforms

Table 2: Comparison of the fold-change data for the 26 miRNA assays common to all three platforms. For each platform, miRNA levels are expressed relative to the control sample A. Two-fold or greater increases in miRNA levels relative to sample A are in bold; two-fold or greater decreases in miRNA levels compared to sample A are in italics. 


\begin{tabular}{|c|c|c|c|c|}
\hline \\
\hline NanoString & $\%$ CV-A & $\% C V-B$ & \multicolumn{2}{|c|}{ Inter-platform Variation Analysis } \\
\hline Mean \%CV & $16.5 \%$ & $17.7 \%$ & $12.2 \%$ & $22.0 \%$ \\
\hline Avg & & & & $17.1 \%$ \\
\hline Qiagen & $\%$ CV-A & $\%$ CV-B & $\%$ CV-C & $\% C V-D$ \\
\hline Mean \%CV & $2.6 \%$ & $5.6 \%$ & $4.3 \%$ & $4.7 \%$ \\
\hline Avg & & & & $4.3 \%$ \\
\hline WaferGen & $\%$ CV-A & $\%$ CV-B & $\%$ CV-C & $\% C V-D$ \\
\hline Mean \%CV & $0.5 \%$ & $0.5 \%$ & $0.5 \%$ & $0.4 \%$ \\
\hline Avg & & & & $0.5 \%$ \\
\hline
\end{tabular}

Table 3: Comparison of $\%$ coefficient of variation for the 26 miRNA assays common to all three platforms, for each Sample A-D. For the NanoString platform, these involve variations in the measured hybridization fluorescent values. For the Qiagen and WaferGen platforms, these depict the variation in the measured $\mathrm{C}_{\text {, }}$ values.

\begin{tabular}{|l|c|c|}
\hline Platform & $\begin{array}{c}\text { Number of Assays with } \\
\text { Fold Change } \mathbf{2} \text { 2 }\end{array}$ & Dynamic Range \\
\hline NanoString & 46 & $0-6.5$ \\
\hline Qiagen & 170 & $0-50.9$ \\
\hline WaferGen & 262 & $0-10,420$ \\
\hline
\end{tabular}

\section{Dynamic Range Analysis}

Table 4: Dynamic range for NanoString, Qiagen and WaferGen assays that displayed a greater than two-fold difference in miRNA levels relative to the control sample.

\begin{tabular}{|l|l|c|c|c|}
\hline & & NanoString & Qiagen & WaferGen \\
\hline HPDE & miR-29b & - & - & - \\
\hline & miR-720 & 1.71 & - & - \\
\hline 8988T & miR-29b & 2.24 & 2.46 & - \\
\hline & miR-720 & - & 3.12 & 2.18 \\
\hline
\end{tabular}

\section{Fold-change Data for miRNAs $29 \mathrm{~b}$ and 720}

Table 5: Comparison of the fold-change data for miRNA assays common to at least two platforms that displayed at least a two-fold increase in levels as compared to untreated HPDE and 8988T cells.

\begin{tabular}{|l|l|c|c|c|}
\hline & & NanoString & Qiagen & WaferGen \\
\hline HPDE & miR-30a & 0.64 & 0.66 & 0.76 \\
\hline 8988T & miR-30a & - & - & - \\
\hline
\end{tabular}

\section{Fold-change Data for miRNA 30a}

Table 6: Comparison of fold-change data for miRNA assays common to all three platforms that displayed at least a two-fold decrease in levels as compared to untreated HPDE cells.

miR-29b in $8988 \mathrm{~T}$ cells was observed with the WaferGen miRNA platform following chloroquine treatment (Table 5).

MiR-720 following chloroquine-autophagy inhibition exhibited a similar induction in expression levels to miR-29b, with all three platforms displaying enhanced miR-720 levels. According to the NanoString data, miR-720 was induced in HPDE cells grown in chloroquine. The fold induction was 1.71 , slightly below the greater than 2-fold cut-off implemented to filter out likely significant miRNA expression changes (Table 5). Utilizing the Qiagen platform, however, miR-720 exhibited a 3.12 fold increase in $8988 \mathrm{~T}$ cells exposed to chloroquine as compared with untreated $8988 \mathrm{~T}$ cells (Table 5). With the WaferGen platform, a 2.18 fold induction of miR-720 was observed in $8988 \mathrm{~T}$ cells exposed to chloroquine as compared with untreated $8988 \mathrm{~T}$ cells (Table 5).

We observed that miR-30a, a known and potent inhibitor of autophagy $[1,14]$, was down-regulated in HPDE cells treated with chloroquine for all three of the miRNA platforms (Table 6). This downregulation was not greater than 2 -fold lower than control levels.

\section{Discussion}

A comparison of the NanoString, Qiagen, and WaferGen miRNA platforms showed that in general, for assays that were common to them, all three platforms behaved similarly in terms of identifying assays that had miRNA levels greater than 2 -fold different in the experimental samples when compared to the control sample, both in magnitude and direction of induction. Of the three platforms, the WaferGen miRNA platform outperformed the other two in terms of reproducibility of the 26 assays in common to all of them, displaying the smallest \% coefficient of variation (\%CV). The Qiagen platform, although also RT-PCR based, displayed an intermediate \%CV whereas the highest $\% \mathrm{CV}$ and therefore the lowest reproducibility was exhibited by the hybridization-based platform, NanoString. From these experiments it can be concluded that RT-PCR based technologies provide higher inter-platform reproducibility than hybridization-based technologies.

Our studies also demonstrated that the WaferGen miRNA platform displayed a dramatically broader dynamic range than the other two platforms for assays that presented a greater than 2-fold induction in miRNA levels relative to the control sample. Again the Qiagen platform, the other real-time PCR based platform, exhibited an intermediate dynamic range. The hybridization based NanoString platform had the smallest dynamic range. The number of assays that met the fold induction criteria and were therefore analyzed for dynamic range were greater for the WaferGen and Qiagen platforms than for the NanoString platform but this difference cannot account for the magnitude of induction observed with the WaferGen platform. Based on the dynamic range data, it can be concluded that the WaferGen platform provides the most sensitive miRNA expression data of the three platforms.

Although the NanoString platform performed poorly compared to the other two platforms in all the inter-platform analyses, the NanoString platform scored highest in our intra-platform variation analysis. The $\mathrm{R}^{2}$ value calculated for the NanoString platform was the highest among all three platforms, reflecting a more linear response and the least variation between triplicate samples. For the Qiagen and WaferGen platforms the $\mathrm{R}^{2}$ values were similar, demonstrating their real-time PCR commonality.

Our cross-platform analysis, in addition to providing valuable information about miRNA expression systems, resulted in several new scientific discoveries regarding miRNA expression and the inhibition of autophagy in normal HPDE cells versus immortalized human PDAC cells. In this manuscript we present the first evidence of miR-720 playing a role in pancreatic ductal adenocarcinoma. We observed a greater than two-fold increase in miR-720 expression in $8988 \mathrm{~T}$ cells grown in the presence of the autophagy inhibitor chloroquine as compared with untreated $8988 \mathrm{~T}$ cells. miR-720 has been shown to be deregulated in various cancers other than pancreatic cancer such as cutaneous malignant melanoma [15], multiple myeloma [16], esophageal cancer [17], vestibular schwannomas [18], and inflammatory breast cancer [19], among others. It has been observed previously that levels of miR-720 and three other miRNAs are significantly higher in biopsies from colorectal cancer patients than normal controls [20]. miR-720 levels are also higher in colorectal patients with mutated KRAS than in those with wild-type genotypes [20]. This could be a downstream effect of ERK pathway over-activation triggered by KRAS mutations. Kimmelman et al. have shown that glutamine supports pancreatic cancer growth through a Kras-regulated metabolic pathway [21,22].

Additionally, we have confirmed previous reports of an association 
of miR-29b production with pancreatic cancer [23,24]. In $8988 \mathrm{~T}$ cells treated with chloroquine, we observed a greater than two-fold induction of miR-29b as compared with untreated $8988 \mathrm{~T}$ cells. It was shown previously that miR-29b was up-regulated greater than 2 fold in two human pancreatic cancer cell lines MiaPACA-2 and PANC-1 that were treated with two chromatin-modifying agents [23]. Previous studies also revealed that the knockdown of Dicer 1 expression in BxPC-3 cells from xenograft pancreatic tumor models and PANC-1 cells resulted in a significant decrease in miR-29b and other miRNAs. It was also shown in the same study, however, that the knockdown of Dicer expression in Capan-2 cells, also from xenograft pancreatic tumor models, had no effect on miR-29b expression but did decrease the expression of other miRNAs [24]. The observation that different pancreatic cancer cell lines exhibit obvious discrepancies in gene expression profiling implies that different molecular mechanisms are involved in the carcinogenesis of pancreatic cancer subclasses and highlights the importance of personalized therapy.

MiR-30a has been shown to be a potent inhibitor of autophagy by down-regulating the expression of Beclin 1 and ATG5, two proteins important in the canonical starvation-induced macroautophagy pathway [25]. It has also been shown that the BCR-ABL tyrosine kinase inhibitor, imatinib, markedly inhibits the expression of miR-30a in human chronic myelogenous leukemia cells [26]. Our finding that miR-30a was down-regulated (although not greater than 2-fold lower than control levels) in the HPDE cells exposed to chloroquine and that this down-regulation was observed for all three platforms may indicate that miR-30a not only plays a role in the inhibition of autophagy but may also play a role in Ras-driven cancers, a correlation that has not been previously documented.

\section{Conclusions}

Based on our findings, miRNA expression analysis can be accomplished using any of these three platforms in a very reproducible manner. The utilization of RT-PCR-based miRNA technologies from Qiagen and WaferGen, however, resulted in higher inter-platform reproducibility and greater sensitivity indicated by larger dynamic range values. The hybridization-based miRNA expression system from NanoString exhibited the best intra-platform reproducibility and had the capability of processing the largest number of samples during a single run, although the sample processing time was the longest of the three platforms, over twenty-four hours. In addition to supplying essential information about miRNA expression systems, our cross-platform analysis enabled us to present this first report of miRNAs involved in the inhibition of autophagy in pancreatic ductal adenocarcinomas. Additional validation and functional analysis can be performed to verify miR-720 and miR-29b as critical biomarkers of response to antiautophagy therapies in pancreatic ductal adenocarcinomas. Also, the exact role of miR-30a which plays a role in the inhibition of autophagy in normal human pancreatic ductal epithelial cells can be explored further. The combination of miRNA manipulation and anti-autophagy treatment with chloroquine should provide new therapeutic avenues for pancreatic ductal adenocarcinoma in the near future.

\section{Acknowledgments}

The authors wish to thank Dr. Alec C. Kimmelman and Dr. Shenghong Yang from the Department of Radiation Oncology at Harvard Medical School for providing the RNA samples for this study. The authors would also like to thank Dr. Winston Patrick Kuo for providing access to the miRNA platforms. The authors also thank the vendors NanoString, Qiagen, and WaferGen for supplying the assays and reagents required for this study. The authors declare neither conflicts nor financial interests. The content is solely the responsibility of the authors and does not necessarily represent the official views of the Harvard Catalyst, Harvard University and its affiliated academic health care centers.

\section{References}

1. Frankel LB, Lund AH (2012) MicroRNA regulation of autophagy. Carcinogenesis 33: $2018-2025$.

2. Tripathi DN, Chowdhury R, Trudel LJ, Tee AR, Slack RS, et al. (2013) Reactive nitrogen species regulate autophagy through ATM-AMPK-TSC2-mediated suppression of mTORC1. Proc Natl Acad Sci USA 110: E2950- E2957.

3. Yang S, Wang X, Contino G, Liesa M, Sahin E, et al. (2011) Pancreatic cancers require autophagy for tumor growth. Genes Dev 25: 717-729.

4. Gilbert K, Mishra G (2006) Pancreatic Cancer: Epidemiology and Pathology Practical Gastroenterology 22-32.

5. Rubensztein DC, Gestwicki JE, Murphy LO, Klionsky DJ (2007) Potential therapeutic applications of autophagy. Nat Rev Drug Discov 6: 304-312.

6. Wolpin BM, Rubinson DA, Wang X, Chan JA, Cleary JM, et al. (2014) Phase II and pharmacodynamic study of autophagy inhibition using hydroxychloroquine in patients with metastatic pancreatic adenocarcinoma. Oncologist 19: 637638.

7. Kolbert CP, Feddersen RM, Rakhshan F, Grill DE, Simon G, et al. (2013) Multiplatform analysis of microRNA expression measurements in RNA from fresh frozen and FFPE tissues. PLoS One 8: e52517.

8. Griffiths-Jones S (2004) The microRNA Registry. Nucleic Acids Res 32: 109-111.

9. Griffiths-Jones S, Grocock RJ, van Dongen S, Bateman A, Enright AJ (2006) miRBase: microRNA sequences, targets and gene nomenclature. Nucleic Acids Res $34: 140-144$

10. Griffiths-Jones S, Saini HK, van Dongen S, Enright AJ (2008) miRBase: tools for microRNA genomics. Nucleic Acids Res 36: 154-158.

11. Kozomara A, Griffiths-Jones S (2011) miRBase: integrating microRNA annotation and deep-sequencing data. Nucleic Acids Res 39: 152-157.

12. Livak KJ, Schmittgen TD (2001) Analysis of relative gene expression data using real-time quantitative PCR and the 2(-Delta Delta C(T)) Method. Methods 25: 402-408.

13. Mestdagh $P$, Van Vlierberghe $P$, De Weer $A$, Muth $D$, Westermann $F$, et al. (2009) A novel and universal method for microRNA RT-qPCR data normalization. Genome Biol 10: R64.

14. Zhu H, Wu H, Liu X, Li B, Chen Y, et al. (2009) Regulation of autophagy by a beclin 1-targeted microRNA, miR-30a, in cancer cells. Autophagy 5: 816-823.

15. Sand M, Skrygan M, Sand D, Georgas D, Gambichler T, Hahn SA, et al. (2012) Comparative microarray analysis of microRNA expression profiles in primary cutaneous malignant melanoma, cutaneous malignant melanoma metastases, and benign melanocytic nevi. Cell Tissue Res 351: 85-98.

16. Jones Cl, Zabolotskaya MV, King AJ, Stewart HJ, Horne GA, et al. (2012) Identification of circulating microRNAs as diagnostic biomarkers for use in multiple myeloma. Br J Cancer 107: 1987-1996.

17. Shinozuka E, Miyashita M, Mizuguchi Y, Akagi I, Kikuchi K, et al. (2012) SnoN/ SKIL modulates proliferation through control of hsa-miR-720 transcription in esophageal cancer cells. Biochem Biophys Res Commun 430: 101-106.

18. Torres-Martin M, Lassaletta L, de Campos JM, Isla A, Gavilan J, et al. (2013) Global Profiling in Vestibular Schwannomas Shows Critical Deregulation of MicroRNAs and Upregulation in Those Included in Chromosomal Region 14q32. PLoS One 8: e65868.

19. Lerebours F, Cizeron-Clairac G, Susini A, Vacher S, Mouret-Fourme E, et al (2013) miRNA expression profiling of inflammatory breast cancer identifies a 5-miRNA signature predictive of breast tumor aggressiveness. Int $\mathrm{J}$ Cancer 133: $1614-1623$

20. Ragusa M, Statello L, Maugeri M, Majorana A, Barbagallo D, et al. (2012) Specific alterations of the microRNA transcriptome and global network structure in colorectal cancer after treatment with MAPK/ERK inhibitors. J Mol Med (Berl) 90: 1421-1438.

21. Ying H, Kimmelman AC, Lyssiotis CA, Hua S, Chu GC, et al. (2012) Oncogenic Kras maintains pancreatic tumors through regulation of anabolic glucose metabolism. Cell 149: 656-670.

22. Son J, Lyssiotis CA, Ying H, Wang X, Hua S, et al. (2013) Glutamine supports pancreatic cancer growth through a KRAS-regulated metabolic pathway. Nature 496: 101-105. 
Citation: Sardi SH, Glassner BJ, Chang JR, Yim SH (2014) Identification of Critical Biomarkers Responsive to Anti-Autophagy Therapies for Pancreatic Ductal Adenocarcinoma through a Performance Analysis of miRNA Platforms. J Bioanal Biomed S10: 001. doi:10.4172/1948-593X.S10-001

23. Lee KH, Lotterman C, Karikari C, Omura N, Feldmann G, et al. (2009) Epigenetic silencing of MicroRNA miR-107 regulates cyclin-dependent kinase 6 expression in pancreatic cancer. Pancreatology 9: 293-301.

24. Wang X, Zhao J, Huang J, Tang H, Yu S, Chen Y (2012) The regulatory roles of miRNA and methylation on oncogene and tumor suppressor gene expression in pancreatic cancer cells. Biochem Biophys Res Commun 425: 51-57.
25. Yu Y, Cao L, Yang L, Kang R, Lotze M, Tang D (2012) microRNA 30A promotes autophagy in response to cancer therapy. Autophagy 8: 853-855.

26. Yu Y, Yang L, Zhao M, Zhu S, Kang R, Vernon P, et al. (2012) Targeting microRNA-30a-mediated autophagy enhances imatinib activity against human chronic myeloid leukemia cells. Leukemia 26: 1752-1760.
This article was originally published in a special issue, Mechanisms and Gene Regulation: Normal and Pathogenomics handled by Editor. Dr. Carl Edwards, Sichuan University, China 